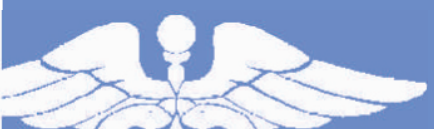

ISSN: 2782-7550 (Print) ISSN: 2782-7542 (Online)
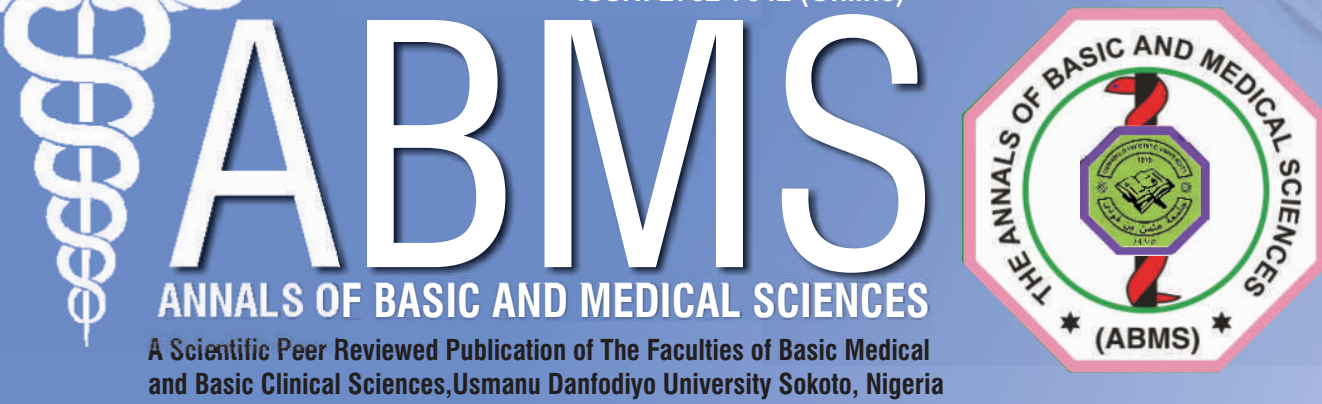

and Basic Clinical Sciences,Usmanu Danfodiyo University Sokoto, Nigeria
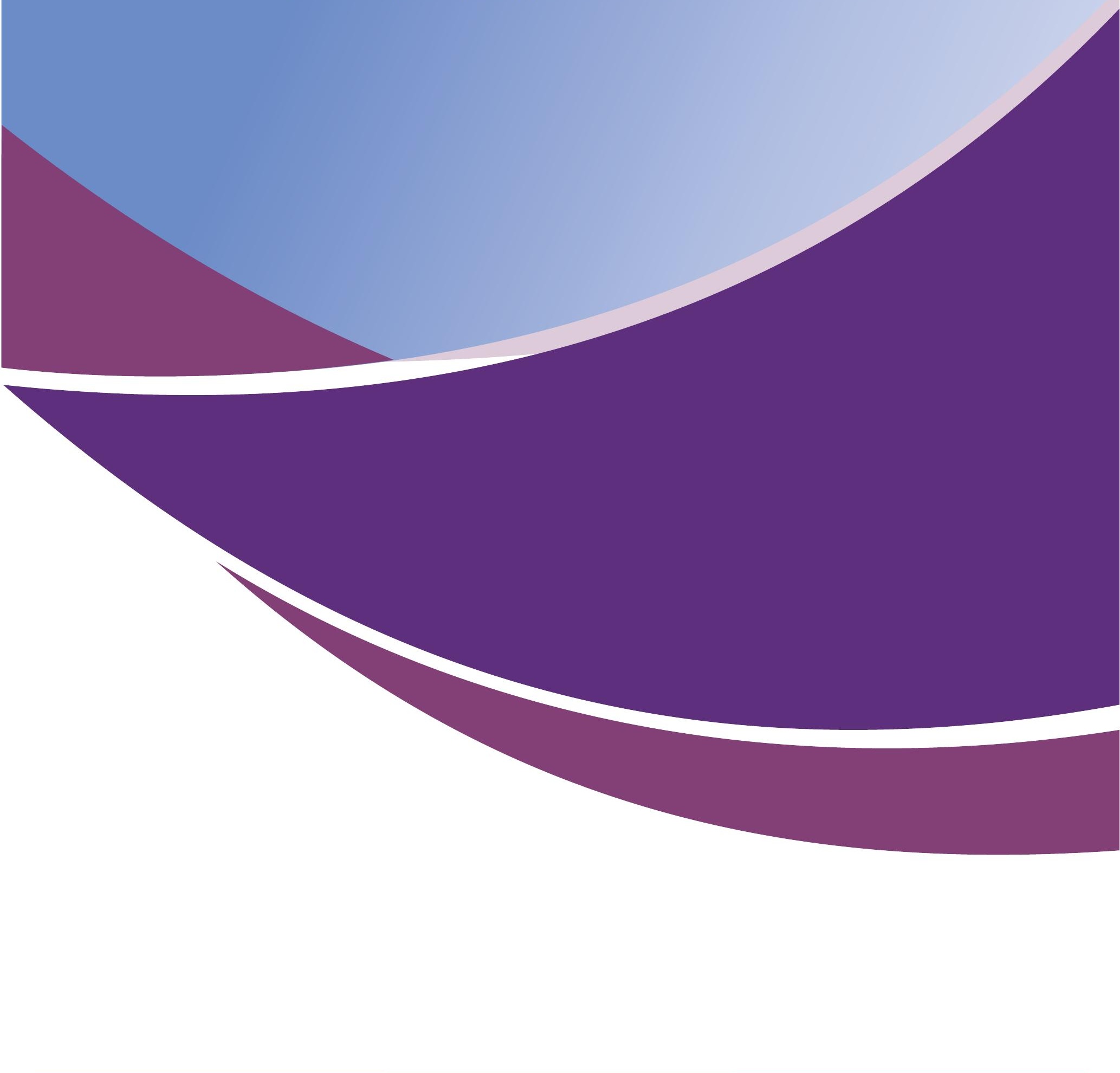


\title{
Risk of Anastrozole-related Mood Disturbances and Dizziness among Breast Cancer Patients taking Anastrozole
}

\author{
Murtala B. Abubakar ${ }^{1 *}$, Venkata Murali Krishna Bhavarajuㄹ, Asrenee Ab Razak ${ }^{3}$ \& Siew Hua Gan ${ }^{4}$ \\ ${ }^{1}$ Department of Physiology, Faculty of Basic Medical Sciences, College of Health Sciences, Usmanu Danfodiyo \\ University PMB 2254 Sokoto, Nigeria. \\ ${ }^{2}$ Department of Nuclear Medicine, Radiotherapy and Oncology
}

${ }^{3}$ Department of Psychiatry, School of Medical Sciences, Universiti Sains Malaysia,16150 Kota Bharu Kelantan ${ }^{4}$ School of Pharmacy, Monash University Malaysia, Jalan Lagoon Selatan, 47500 Bandar Sunway, Selangor, Malaysia

\section{Abstract:}

Background: Anastrozole has been widely utilized in the management of endocrine sensitive post-menopausal breast cancer; however, there is high variability in the adverse reactions associated with its use. Anastrozole-associated mood disturbances and dizziness occur less frequently. However, they can still affect the quality of life of breast cancer patients. The aim of this study was to determine the clinical and demographic factors associated with Anastrozole's adverse events.

Materials/Methods: This is a cross-sectional study of oestrogen receptor positive post-menopausal women $(n=97)$ with stages I to III breast cancer receiving Anastrozole (1mg daily). Multivariate analyses were performed to establish the factors associated with Anastrozole-induced mood disturbance and dizziness.

Results: Approximately $20.6 \%$ and $13.4 \%$ of the subjects experienced mood disturbance and dizziness respectively. Patients who are on Anastrozole treatment for more than three years had higher odds of having mood disturbance (adjusted odds ratio 20.31, confidence interval 1.75 to 235.31, $p=0.016$ ). No significant association was established between serum oestrogen levels and development of mood disturbance and dizziness.

Conclusion: Our study confirmed that although mood disturbances and dizziness are not the most commonly reported adverse reactions, the duration of Anastrozole treatment may be a predictor of mood disturbance in these patients.

Keywords: Anastrozole, mood disturbance, dizziness, post-menopausal, breast cancer.

Corresponding author: Murtala B. Abubakar Department of Physiology, Faculty of Basic Medical Sciences, College of Health Sciences, Usmanu Danfodiyo University PMB 2254 Sokoto, Nigeria. E-mail: murtala.bello@udusok.edu.ng Tel: +234 8035925103

\section{Introduction}

0 estrogens play a significant role in breast cancer progression and on the basis of presence of oestrogen receptors, breast cancers are classified as hormone receptor positive or negative. Approximately $75 \%$ of all breast cancer patients are hormone receptor positive and their tumour growth is dependent on the presence of oestrogens (1). Until recently, tamoxifen (a selective oestrogen receptor blocker) has been the drug of choice in the adjuvant therapy of both pre- and post-menopausal oestrogen receptor positive breast cancer (2, 3). However, current guidelines recommend that Anastrozole or any of the other two types of aromatase inhibitors such as exemestane or letrozole should be used as alternative to tamoxifen following three to five years of treatment with tamoxifen (4). A number of trials have demonstrated the benefit of using aromatase inhibitors as adjuvant in the treatment of post-menopausal hormone receptor positive earlyand advanced-stage breast cancers $(5,6)$. Additionally, treatments with Anastrozole and tamoxifen have been compared either when administered singly or in combination in a clinical trial (ATAC trial) which has demonstrated that the former is more efficacious or is less toxic (7).

The source of oestrogen in a woman varies markedly depending on the menopausal status. In pre-menopausal woman, the main source of oestrogen is the ovary, whereas in post-menopausal woman, it is produced from various sources including the adipose tissue, breast, brain, liver and the muscles $(8,9)$. The key enzyme involved in the biosynthesis of oestrogen in the aforementioned extra-gonadal sources is the aromatase, which catalyses the conversion of androstenedione to oestrogen (8). Anastrozole acts by blocking the conversion of androgens into estrone and oestradiol by the aromatase enzyme (10).

Low circulating levels of oestrogen in women are linked to pre-menstrual syndrome, postnatal depression and post-menopausal depression (11). Interestingly, administration of Anastrozole at $1 \mathrm{mg}$ daily has been reported to completely suppress the circulating oestrogen levels [12]. In 
general, the adverse effects resulting from treatment with Anastrozole and other aromatase inhibitors are more manageable when compared to those from tamoxifen $(7,13)$. However, despite the superiority of Anastrozole over tamoxifen, a significant number of patients experience adverse effects such as musculoskeletal complaints, vaginal dryness or dyspareunia, hot flashes, mood disturbance and dizziness (14-17).

Among the three types of aromatase inhibitors currently in use, Anastrozole is associated with a higher incidence of mood changes than the other two (16). For example, there was an evidence from two case reports of Anastrozole-associated mood disturbances (16). In the ATAC trial, it was shown that Anastrozole was associated with a higher percentage of mood disturbances (19.3\%) when compared to tamoxifen (17.9\%) (18). It has also been reported that treatment of breast cancer patients with Als such as anastrozole is not associated with increased risk of depressive symptoms (19) and is even associated with improvement of depressive symptoms in early breast cancer patients (20). Nevertheless, to our knowledge, no study has investigated the association between sociodemographic factors and Anastrozole-related mood changes and dizziness. Therefore, the objective of the present study is to investigate the relationship between these adverse events and socio-demographic and clinical variables.

\section{Materials and Methods}

\section{Study population}

This was a cross-sectional study of 94 postmenopausal women (aged between 44 and 83 years) with oestrogen receptor positive breast cancer who attended the Department of Nuclear Medicine, Radiotherapy and Oncology clinic, Universiti Sains Malaysia, Kelantan, Malaysia between November 2014 and August, 2015. The protocol was approved by the Human Research Ethical Committee of the Universiti Sains Malaysia (USMKK/PPP/JEPeM [260.3.(21)]) which complies with the Declaration of Helsinki. The subjects were post-menopausal women with histologically confirmed hormone receptor positive stages I to III breast cancer based on the American Joint Committee on Cancer (AJCC) staging manual (sixth Edition). All patients received $1 \mathrm{mg} / \mathrm{day}$ of Anastrozole. Patients who were previously on tamoxifen but changed to Anastrozole (for at least four weeks) at the time of enrolment were also included in the study. However, those who were taking hormone replacement therapy (HRT), had an underlying psychiatric illness, chronic liver or renal diseases were excluded. Following screening of the medical records, the patients were then approached for study enrolment at their regular follow-up appointments. Only patients who signed written informed consents were enrolled following which a case report form was individually completed. Peripheral blood $(3 \mathrm{ml})$ was collected for hormonal assay (follicle stimulating hormone, FSH; Luteinizing hormone, LH and oestradiol, E2).

\section{Clinical and demographic data}

As mood disturbances in these subjects may also be attributed to other causes, we instructed the participants to characterise the causes of their mood disturbance through a pro forma modified from the mood rating scale (21) which has been previously used in the assessment of effects of cancer therapy and assessment of endocrine therapy-related hot flashes and mood disturbances $(22,23)$. The reported mood disturbances may include any of these symptoms such as inner tension, despondency, tearfulness, nervousness, aggressiveness and lack of drive.

Patients demographic variables, such as age, race, marital status, the number of children, occupational status, educational level, age of menopause, years since menopause, age at time of diagnosis of breast cancer, family history of breast cancer and history of contraceptive use were ascertained. Other variables such as weight and height were recorded at the clinic during the routine follow up visit. Clinical variables, such as cancer stage, tumour grade, human epidermal growth factor (HER2) status, current Anastrozole use, time since Anastrozole was commenced and number of comorbidities were also derived from the patient's case folder and then verified by an oncologist for quality control.

\section{Laboratory data}

The latest routine laboratory reports of liver and kidney functions were ascertained from the patients' medical record.

\section{Laboratory Analysis}

Serum E2 and follicle stimulating hormone (FSH) levels were measured using one- and two-steps quantitative immuno assay (using chemiluminescent microparticle) technologies respectively, with flexible assay protocols referred to as chemiflex.

\section{Statistical analysis}

Data analysis was performed using IBM SPSS Statistics (Version 22.0. Armonk, NY: IBM Corp). A simple logistic regression between each of the two outcomes (dependent variables, mood disturbance and dizziness) and all the covariates mentioned above was conducted.

\section{Results}

\section{Patient characteristics}

A total of 97 patients aged between 44 to 83 years, with a mean age of 58.1 (SD \pm 7.3 ) were screened for the study (Table 1). Three of the subjects were unsure whether their symptoms were associated with Anastrozole intake and were therefore excluded from the analyses.

\section{Mood disturbances}

Among the study subjects, 20 (20.6\%) reported having experience of mood disturbance (Table 2). Only marital status and duration of Anastrozole treatment were 
significant in the multiple logistic regression analyses. Unmarried patients had higher odds of having mood disturbance with adjusted odds ratio (AOR) of 34.83 [95\% confidence interval $(\mathrm{Cl}) 2.36,514.74, p=0.010$.]

Similarly, patients with more than three years of treatment with Anastozole had increased odds of having mood disturbance (AOR, 20.30, $\mathrm{Cl} ; 1.75,235.31$, $p=0.016)$.

Table 1: Socio-demographic and clinical variables

\begin{tabular}{|c|c|c|}
\hline Variable & $\mathrm{n}$ & $\%$ \\
\hline Age (mean, SD) & 58.1 & 7.3 \\
\hline \multicolumn{3}{|l|}{ Marital status } \\
\hline Married & 90 & 92.8 \\
\hline Not married & 7 & 7.2 \\
\hline \multicolumn{3}{|l|}{ Race } \\
\hline Malays & 76 & 78.4 \\
\hline Chinese & 20 & 20.6 \\
\hline Indians & 1 & 1.0 \\
\hline \multicolumn{3}{|l|}{ Educational status } \\
\hline No formal education & 7 & 7.2 \\
\hline Primary and high school & 72 & 74.2 \\
\hline Tertiary & 14 & 14.4 \\
\hline Unanswered & 4 & 4.1 \\
\hline \multicolumn{3}{|l|}{ Occupational status } \\
\hline Unemployed & 63 & 64.9 \\
\hline Employed & 34 & 35.1 \\
\hline \multicolumn{3}{|l|}{ Blood Pressure (mmHg) (mean, SD) } \\
\hline Systolic & 138.7 & 20.5 \\
\hline Diastolic & 80.8 & 11.4 \\
\hline \multicolumn{3}{|l|}{ Stage } \\
\hline 1 & 10 & 10.3 \\
\hline II & 44 & 45.4 \\
\hline III & 43 & 44.3 \\
\hline \multicolumn{3}{|l|}{ Grade } \\
\hline I & 18 & 18.6 \\
\hline II & 39 & 40.2 \\
\hline III & 25 & 25.8 \\
\hline Unknown & 15 & 15.5 \\
\hline \multicolumn{3}{|l|}{ HER2 status } \\
\hline Negative & 52 & 53.6 \\
\hline Positive & 35 & 36.1 \\
\hline Unknown & 10 & 10.3 \\
\hline Age of menopause (mean, SD) & 50.1 & 3.8 \\
\hline \multicolumn{3}{|l|}{ Years since menopause } \\
\hline$>10$ years & 22 & 23.9 \\
\hline $5-10$ years & 36 & 37.0 \\
\hline$<5$ years & 35 & 34.8 \\
\hline Unsure & 4 & 4.3 \\
\hline Age at diagnosis of breast cancer (mean, SD) & 51.0 & 15.0 \\
\hline \multicolumn{3}{|l|}{ Family history of breast cancer } \\
\hline No & 73 & 79.3 \\
\hline \multicolumn{3}{|l|}{ BMI } \\
\hline$\leq 25$ & 42 & 43.3 \\
\hline$>25$ & 55 & 56.7 \\
\hline \multicolumn{3}{|l|}{ Number of comorbidities } \\
\hline None & 46 & 47.4 \\
\hline 1 & 27 & 27.8 \\
\hline$\geq 2$ & 16 & 16.5 \\
\hline Unconfirmed & 8 & 8.2 \\
\hline \multicolumn{3}{|l|}{ Time since anas } \\
\hline$<1$ year & 52 & 53.6 \\
\hline 1 - 3 years & 34 & 35.1 \\
\hline$>3$ years & 11 & 11.3 \\
\hline FSH (IU/L) (mean, SD) & 59.2 & 22.5 \\
\hline LH (IU/L) (mean, SD) & 24.9 & 10.3 \\
\hline \multicolumn{3}{|l|}{$\mathrm{E} 2(\mathrm{pmol} / \mathrm{L})$} \\
\hline Undetectable & 54 & 55.7 \\
\hline Detectable & 23 & 23.7 \\
\hline Not available & $\begin{array}{l}23 \\
20\end{array}$ & 20.6 \\
\hline
\end{tabular}

SD, standard deviation, HER2, human epidermal growth factor receptor 2 , $\mathrm{BMI}$, body mass index

\section{Dizziness}

Approximately $13.4 \%$ of the subjects reported Anastrozole-associated dizziness. Only marital status was significantly associated with increased odds of having dizziness (AOR, 9.50, Cl, 1.21, 74.27, $p=0.032$ ) (Table 3).

Table 2: Simple logistic regression analyses of factors associated with anas-related mood disturbance

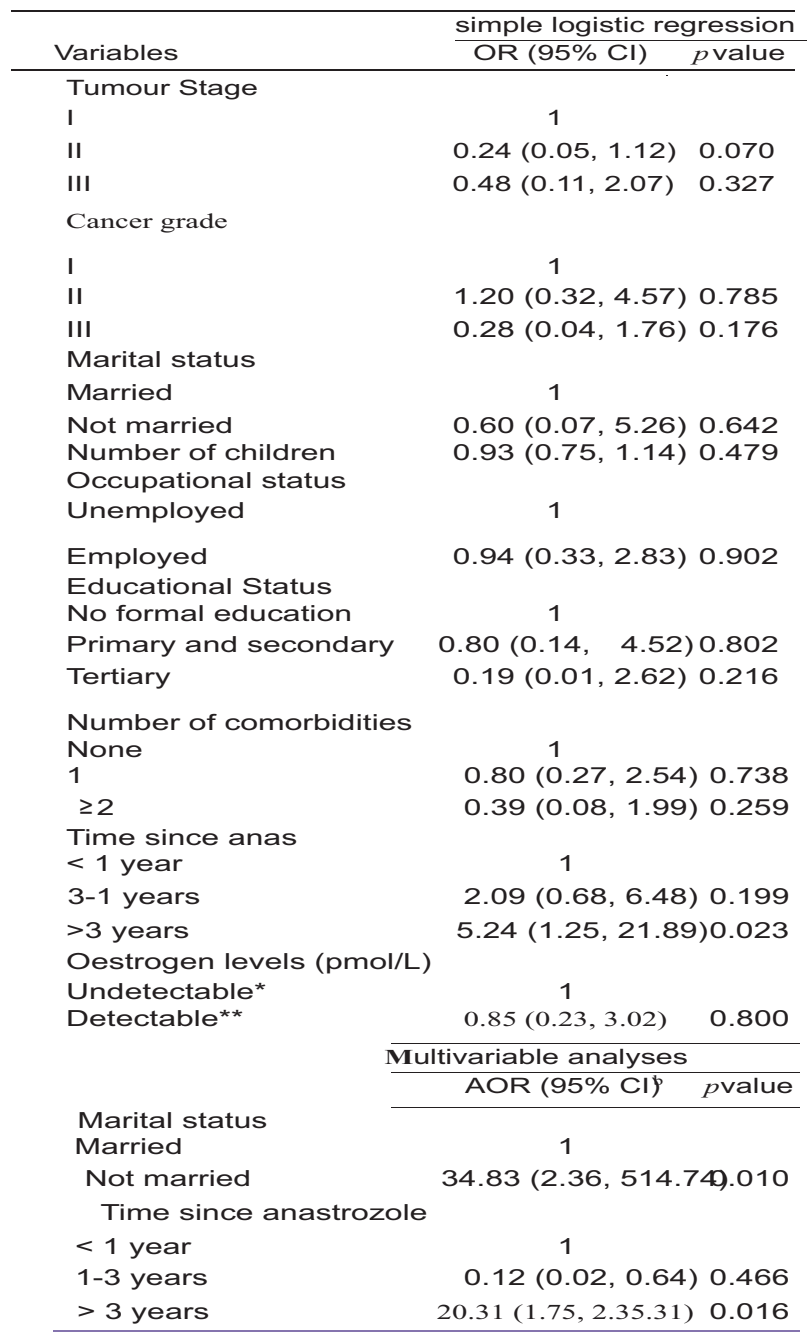

OR, odds ratio, $\mathrm{AOR}$, adjusted $\mathrm{OR}, 95 \% \mathrm{Cl}, 95 \%$ confidence interval ${ }^{*}$ Estrogen level $<36.7 \mathrm{pmol} / \mathrm{L}^{* *}$ Detectable but within normal range (>36.7 to $88.1 \mathrm{pmol} / \mathrm{L}$ ).

\section{Discussion}

The present study reports the association between socio-demographic factors and Anastrozole-related mood changes and dizziness. These adverse events could affect the quality of life of breast cancer patients and their compliance to treatment. As a result of this, investigating the factors associated with these symptoms will provide further guidance in targeted management to improve the quality of life of post-menopausal breast cancer patients receiving Anastrozole treatment.

The percentage of patients (20.6\%) with Anastrozolerelated mood disturbance found in our study is close to the one reported in the ATAC trial at $19.3 \%$ (18). Our study demonstrated that patients receiving Anastrozole for 
more than three years had increased odds of having mood disturbances compared to those who received shorter treatment duration. The reason for this may be attributed to the long-term depletion of E2 levels as a result of Anastrozole treatment since low serum oestrogen levels in women have been linked to mood changes (11). However, in the present study, no significant association was established between patients' serum E2 levels and development of mood disturbances which may be attributed to the small sample size. In addition, the present study did not assess the specific mood symptoms, but assessed the mood disturbances based on the intensity, thus limiting the analysis of specific symptoms of mood disturbance caused by Anastrozole intake. However, we have demonstrated that patients with detectable normal limit of serum E2 levels had reduced odds of developing at least one Anastrozole-associated adverse effect compared to those with completely undetectable E2 concentrations (unpublished data). This may therefore further suggest that E2 suppression may play an important role in mood changes associated with Anastrozole.

Our study found that $13(13.4 \%)$ of the patients experienced dizziness. However, this rate is higher than that seen in clinical trial settings (3.1\%) (17) and may be due to variation in patient versus clinician reported adverse reactions in which symptoms like vomiting and diarrhoea are more easily observable than subjective symptoms such as fatigue and dyspnoea (25). Nevertheless, the impact of sociodemographic and clinical variables on Anastrozole-associated adverse events should be further investigated in a larger cohort.

One of the limitations of this study is the fact that the study samples were assessed for both exposure to the drug and outcome at a single point in time, therefore, it was not demonstrated whether the exposure to the drug preceded the observed disturbance in mood. Another limitation of the study is the absence of a negative control in order to rule out the effects of confounding factors. This is because it has been reported that breast cancer itself is associated with increased risk of developing symptoms of anxiety and depression (20).

\section{Conclusions}

Our study further confirmed that mood disturbances and dizziness are not the most commonly reported adverse reactions among post-menopausal women with oestrogen receptor positive breast cancer receiving Anastrozole. It also suggests that duration of Anastrozole treatment may be a predictor of mood disturbance in breast cancer patients although serum E2 levels may not predict development of mood disturbance and dizziness in Anastrozole treated breast cancer patients.

\section{Acknowledgement}

This work was supported by grant no. 1001/PPSP/ 853005 from Universiti Sains Malaysia (USM).

\section{Conflicts of interest}

There are no conflicts of interest.

\section{References}

1. Kathy.I. Pritchard, K.A. Gelmon, D. Rayson, L. Provencher, M. Webster, D. McLeod, S. Verma. Endocrine therapy for post-meno pausal women with hormone receptor-positive her2-negative adva nced breast cancer after progression or recurrence on nonsteroidal aromatase inhibitor therapy: a Canadian consensus statement. Current Oncology 2013; 20(1): 48-61.

2. Lucy Ann Behan, Eitan Amir, Robert F. Casper. Aromatase inhibitors for prevention of breast cancer in post-menopausal women: a nar rative review. Menopause 2015; 22(3): 342-50.

3. Gaia Schiavon, Ian E. Smith. Status of adjuvant endocrine therapy for breast cancer. Breast Cancer Research 2014; 16(2): 206.

4. NCCN. National Comprehensive Cancer Network Clinical Practice Guidelines in Oncology-Breast Cancer. 2016 18/3/2017]; Version 1. 2012:[Available from: https://www.nccn.org/professionals/physician_ gls/f guidelines.asp.

5. James N. Ingle. Adjuvant endocrine therapy for postmenopausal women with early breast cancer. Clinical Cancer Research 2006; 12(3): 1031s-1036s.

6. James N. Ingle, Vera J. Suman. Aromatase inhibitors for therapy of advanced breast cancer. The Journal of Steroid Biochemistry and Molecular Biology 2005; 95(1): 113-119.

7. John F. Forbes, Jack Cuzick, Aman Buzdar, Anthony Howell, Jeffrey S. Tobias, Michael Baum. Effect of anastrozole and tamoxifen as adjuvant treatment for early-stage breast cancer: 100-month ana lysis of the ATAC trial. The lancet oncology 2008; 9(1): 45-53.

8. E.R. Simpson, M.S. Mahendroo, G.D. Means, M.W. Kilgore, M.M. Hinshelwood, S. Graham-Lorence, B. Amarneh, Y. Ito, C.R. Fisher, M.D. Michael, et al. Aromatase Cytochrome P450, The Enzyme Responsible for Estrogen Biosynthesis. Endocrine reviews 1994; 15(3): 342-355.

9. A.A. van Landeghem, J. Poortman, M. Nabuurs, J.H. Thijssen. Endogenous concentration and subcellular distribution of estrogens in normal and malignant human breast tissue. Cancer Research 1985; 45(6): 2900-2906.

10.Ian E. Smith, Mitch Dowsett. Aromatase inhibitors inbreast cancer. The New England Journal of Medicine 2003; 348(24): 2431-42.

11.George Fink, Barbara E. H. Sumner, Roberta Rosie, Oliver Grace, John P. Quin. Estrogen control of central neurotransmission: effect on mood, mental state, and memory. Cellular and Molecular Neuro biology 1996; 16(3): 325-44.

12.Paul V. Plourde, Martin Dyroff, Michael Dukes. Arimidex®: a potent and selective fourth-generation aromatase inhibitor. Breast cancer research and treatment 1994; 30(1): 103-111.

13.Boccardo , P. Guglielmini, R. Bordonaro, A. Fini, B. Massidda, M. Porpiglia, R. Roagna, P. Serra, L. Orzalesi, G Ucci, A. Rubagotti. Switching to anastrozole versus continued tamoxifen treatment of early breast cancer: long term results of the Italian Tamoxifen Anastrozole trial. European Journal of Cancer 2013; 49(7): 1546 $-1554$.

14. Henning T. Mouridsen. Incidence and management of side effects associated with aromatase inhibitors in the adjuvant treatment of breast cancer in postmenopausal women. Current Medical Research and Opinions 2006; 22(8): 1609-1621.

15. Harold J. Burstein. Aromatase inhibitor-associated arthralgia syn drome. Breast 2007; 16(3): 223-234.

16.Xiomara Rocha-Cadman, Mary Jane Massie, Katherine Du Hamel. Aromatase inhibitors and mood disturbances. Palliative and Sup portive Care 2012; 10(3): 225-227.

17.David Cella, Lesley Fallowfield, Peter Barker, Jack Cuzick, Gershon Locker, Anthony Howell. Quality of life of post-menopausal women in the ATAC ("Arimidex", tamoxifen, alone or in combination) trial after completion of 5 years' adjuvant treatment for early breast cancer. Breast Cancer Research and Treatment 2006; 100(3): 273-284.

18.A. Howell, J. Cuzick, M. Baum, A. Buzdar, M. Dowsett, J.F. Forbes, G. Hoctin-Boes, J. Houghton, G.Y. Locker, J.S. Tobias. Results of the ATAC (Arimidex, Tamoxifen, Alone or in Combination) trial after completion of 5 years' adjuvant treatment for breast cancer. Lancet 2005. 365(9453): 60-62.

19.Bedillion MF, Ansell EB, Thomas GA. Cancer treatment effects on cognition and depression: The moderating role of physical activity. 
The Breast. 2019;44:73-80.

20.Martino G, Catalano A, Agostino RM, Bellone F, Morabito N, Lasco CG, et al. Quality of life and psycho logical functioning in postmeno pausal women undergoing aromatase inhibitor treatment for early breast cancer. PloS one. 2020;15(3):e0230681.

21.J. Anderson, M.B. Walker, V. Swanson, L.G. Walker The Mood Rating Scale: A brief Acceptable, Reliable and Valid State Measure of Normal Mood. Psycho-Oncology 2000; 9(4): 355-364.

22.L.G. Walker, M.B. Walker, J. Anderson, T. Whittaker, E. Simpson, I.C. Smith, S.D. Heys, A.W. Hutcheon,T.K. Sarkar, A.K. Ah-See, et al. Is Quality of Life Better During Primary CVAP or Docetaxel Chemotherapy? Psycho-Oncology 1999; 8(1): 1-13.
23.R. Thomas, M. Williams, C. Marshall, L. Walker Switching to letrozole or exemestane improves hot flushes, mood and quality of life in tamoxifen intolerant women. British Journal of Cancer 2008; 98(9): 1494-1499.

24.S.H. Noor. Multiple Logistic Regression 2016: School of Medical Sciences, Universiti Sains Malaysia.

25.Ethan Basch, Alexia lasonos, Tiffani McDonough, Allison Barz, Ann Culkin, Mark G Kris, Howard I Scher, Deborah Schrag. Patient versus clinician symptom reporting using the National Cancer Institute Common Terminology Criteria for Adverse Events: results of a questionnaire-based study. Lancet Oncology 2006; 7(11): 903-909. 